Primitiveoral reflexesarealwaysaccorded space in neurological textbooks and, excuse the pun, paid lip service to in clinicopathological conferences. The terminology is confusing and the reflexesareoften misinterpreted. They can however, beoccasionally diagnosticallyuseful. There is a profusion of terms in the literature including'snout', 'pout' and 'rooting reflexes', and often inadequate distinction is made between primitivebehavioural responsesand brisk facial myotactic reflexes.

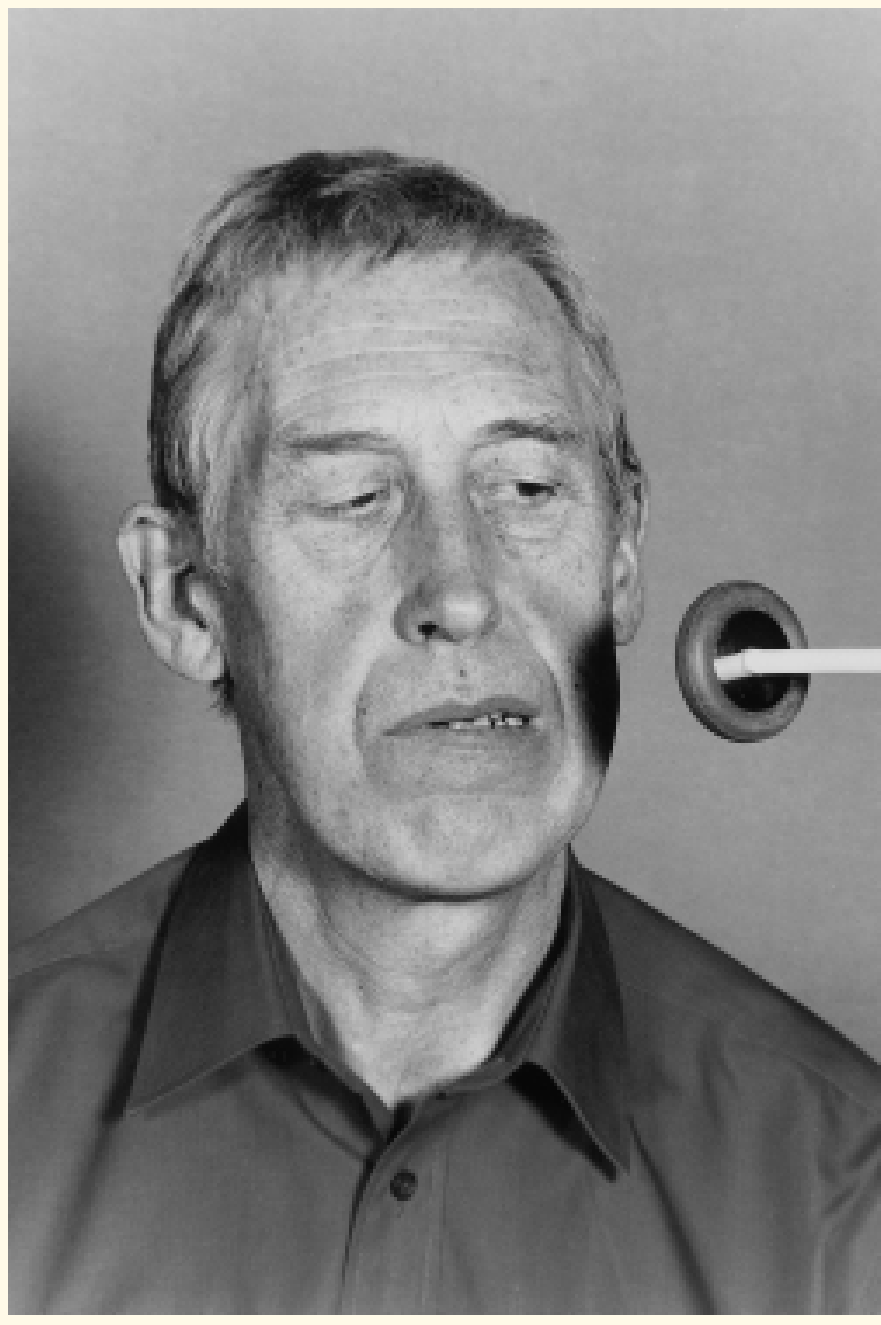

The primitive oral reflexes include sucking and rooting. These are appropriate motor responses in the infant to ensure adequate feeding at the breast. They are subsequently inhibited as the infant matures, but can reemergewith frontal lobedamageor diffusecerebral cortical damage. They may be seen in relative isolation in frontal lobe degenerations and frontal lobe tumours. The sucking reflex, evoked by placing an object such as a spatula in the mouth, is usually only seen in severely demented patients or those with diffuse damage, for example post cerebral anoxia. The sucking reflex is rarely of clinical diagnostic value.

Rootingreflexescan beseen in patients with frontal syndromesand usuallyin thecontext of other frontal reflexes, such as a grasp reflex, instinctive grasp reaction or utilization behaviour. Itisusuallybest to start with the visual rooting reflex. This can be done by asking the patient to look at the head of a tendon hammer, which is brought towards the mouth. Initiallythemouth may open and if the tendon hammer moved to theside, then the lips will turn in that direction (Fig. 1a). One can then go on to elicit a tactile rooting reflex, which may bepresentif thevisual reflex is absent. A finger or the handle of thetendon hammer is drawn across the upper or lower lip in a lateral direction and thelips will moveas if

\section{Martin Rossor}

Dementia Research Group, Department of Clinical Neurology, Institute of Neurology, Queen Square, London, WC1N 3 BG. Email: M.Rossor@dementia.ion. ucl.ac.uk

\title{
Figure $1(\mathrm{a})$
}




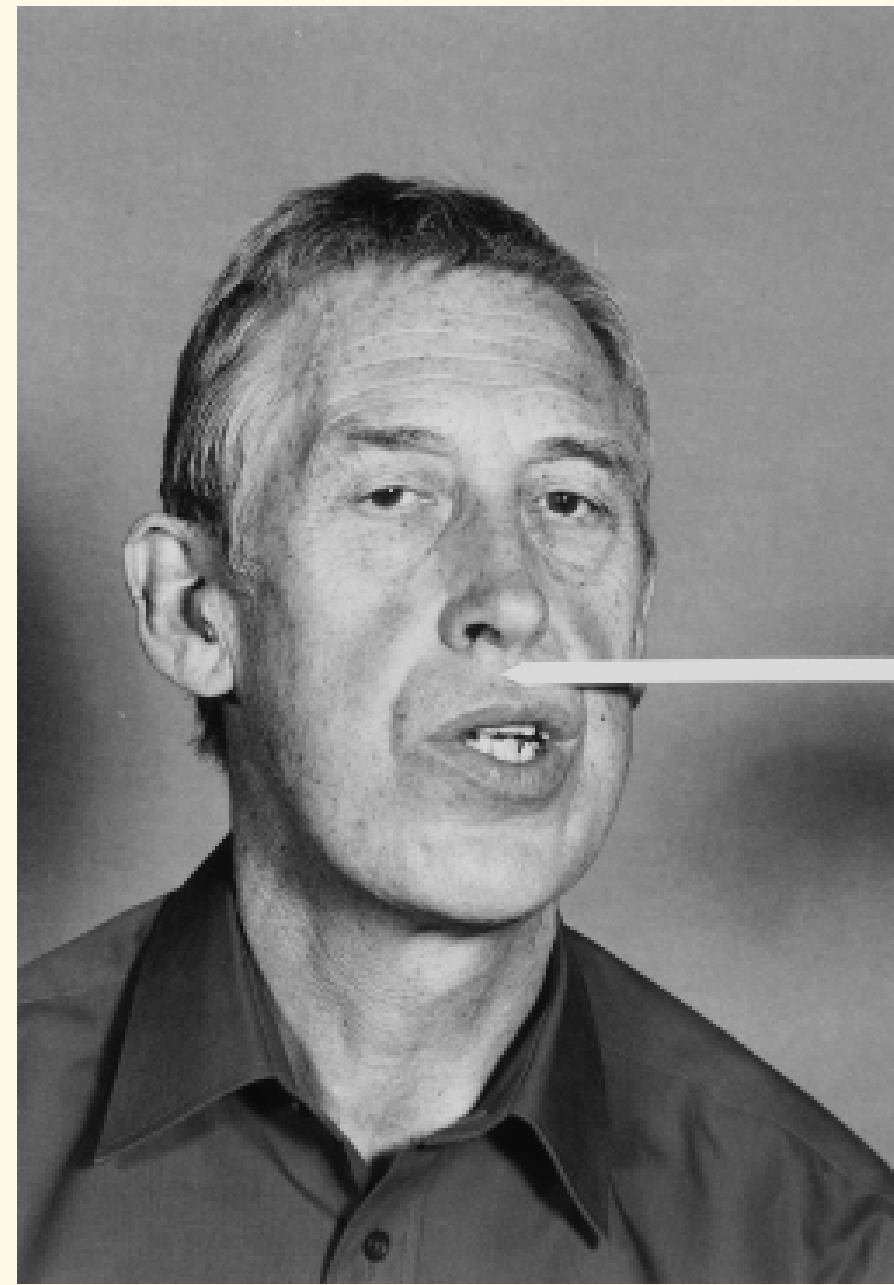

Figure $1(b)$ to take the object in the mouth (Fig. 1b). This movement is slow in response to the stimulus, and physiologically quite distinct from a myotactic reflex of the orbicularis oris, see below (Ekbom et al. 1952).

It is suggested that the snout reflex should really be reserved for the reflex elicited by a constant pressure on the philtrum (Paulson 1977), which results in a puckering of the lips and is essentially a variation of thetactilerooting response (Fig. 2a). These rooting responses can be diagnostically useful in patients in whom apathy is a feature of a frontal lobe syndrome and who have normal neuroimaging. Occasionally one is unsure whether such patients suffer primarily from an affective disorder or an underlying frontal degeneration. The detection of a rooting reflex will direct one towards the latter.

Quite distinct from these primitive behavioural responses is the increased myotactic stretch reflex of the orbicularis oris, which is found in association with other evidence of a brisk facial jerk. This can be elicited by tapping a spatula placed over thelips (Fig. 2b) or by tapping theorbicularis oris directly (Fig. 2C). There is a brisk pouting response, and the term 'pout reflex' is probably best reserved for this brisk facial jerk, indicative of a bilateral upper motor neuronelesion. It ispossiblefor both a pout and rooting reflex to be combined in one patient. Pout reflexes are seen with any bilateral upper motor neurone lesion, such as amyotrophic lateral sclerosis, multiplesclerosis or intracerebral small vessel disease. Patients with a brisk pout reflex dueto small vessel disease often also have a spastic pseudo-smile (Critchley 1986). Small vessel disease is a common comorbidity in the elderly and careful attention to snouting, pouting and rooting can help to elucidate theunderlying cause of cognitiveimpairment.

\section{REFERENCES}

Critchley M (1986) The Citadel of the Senses and Other Essays, pp. 72-3. Raven Press, New York.

Ekbom KA, Jernelius B \& Kugel berg E (1952) Perioral Reflexes. N eurology, 2, 103-11.

Paulson GW (1977) The Neurological Examination in Dementia. In: Dementia, 2 edn (ed. Wells CE), pp. 169-88. FA Davis Co., Philadelphia. 

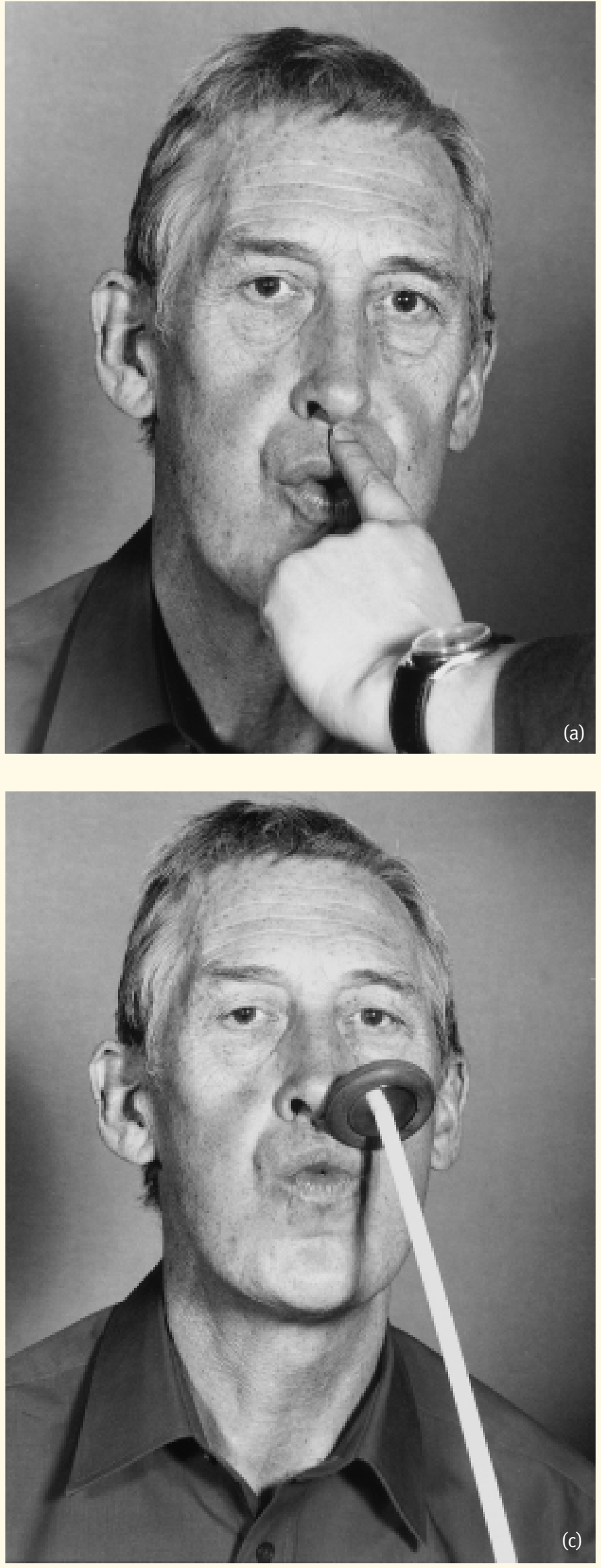

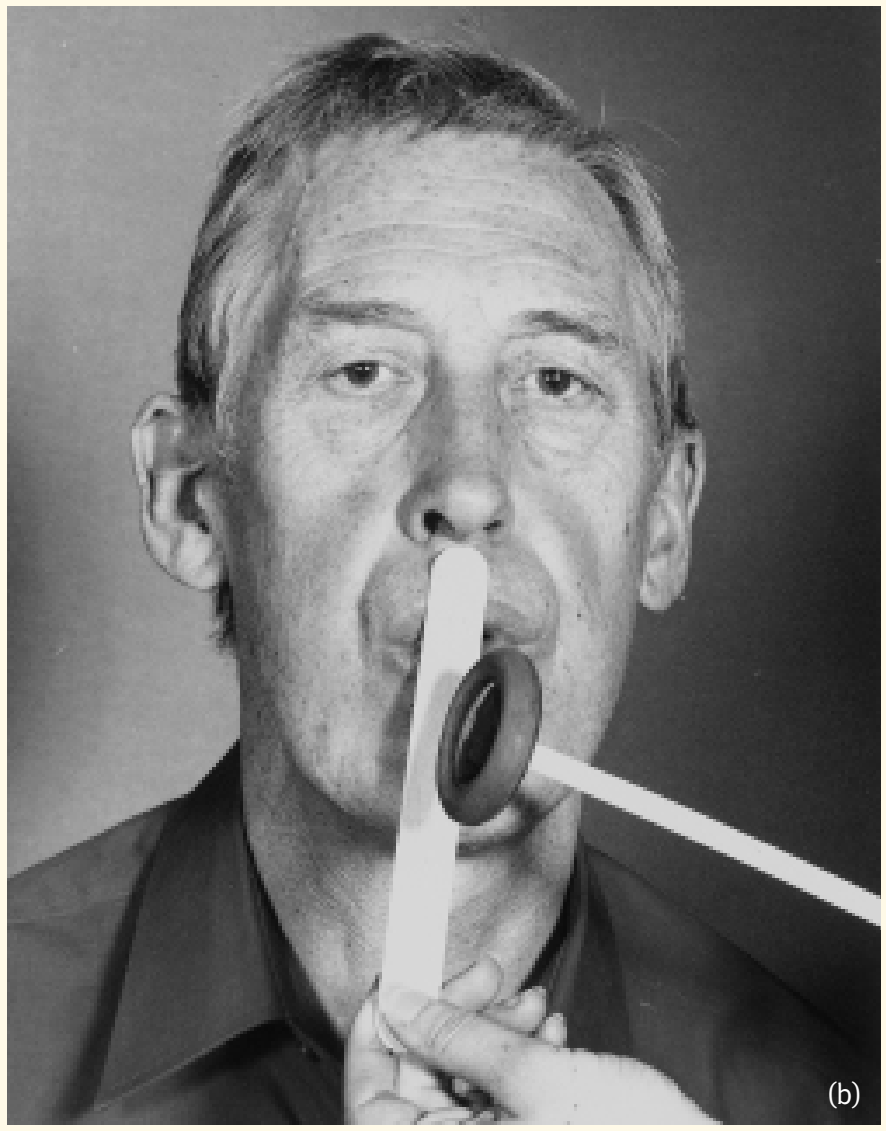

Figure 2

There is a profusion of terms in the literature including 'snout', 'pout' and 'rooting reflexes', and often inadequate distinction is made between primitive behavioural responses and brisk facial myotactic reflexes 